\title{
Green synthesis of copper oxide nanoparticles for biological applications.
}

\author{
*Bhuvaneshwari V, Vaidehi D, Logpriya S \\ PG and Research Department of Biotechnology, Kongunadu Arts and Science College, \\ Coimbatore, Tamil Nadu, India
}

\begin{abstract}
In recent years, scientific community has an interest on nanometer sized materials with unique physical, chemical, and biological properties. Nano-copper oxide is very interested because of their potential applications in many fields such as heterogeneous catalysts, anti-microbial, antioxidants, imaging agents and drug delivery agents in field of biomedicine. Nanoparticles (NPs) can interact with biomolecules and it has useful in diagnosis and treatment cancer. Green synthesized $\mathrm{CuO}$ NPs have being used as photocatalyst, enhanced the rates of textile dye decolourisation, anti-microbial agent and better anti-cancer compound with minimal side effects. $\mathrm{CuO}$ NPs can also act as efficient anti-bacterial agent when incorporated in coatings, plastics, textiles, etc.
\end{abstract}

Accepted on December 14, 2017

\section{Editorial}

In recent years, scientific community has an interest on nanometer sized materials with unique physical, chemical, and biological properties [1]. Copper oxide $(\mathrm{CuO})$ is broadly used with wide range of applications in number of fields such as catalyst, sensing materials, ceramics, thermoelectric materials, glass, superconducting materials and anti-microbial activity [2]. Nano-copper oxide is very interested because of their potential applications in many fields such as heterogeneous catalysts, anti-microbial, anti-oxidants, imaging agents and drug delivery agents in field of biomedicine [3]. Usually nanostructures ranges from 1 to $100 \mathrm{~nm}$ in size compared to large biological molecules such as enzymes, receptors etc. Nanoparticles (NPs) can interact with biomolecules and it has useful in diagnosis and treatment cancer $[4,5]$.

Data related to anti-microbial activity of CuO NPs is very less compared to other metal nanoparticles [6,7]. These nanoparticles are very stable, and their activity is longer when compared with organic anti-microbial agents. Synthesis of $\mathrm{CuO}$ NPs is very less expensive when compared to gold and silver nanoparticles whish possess excellent anti-microbial agent because of their unusual crystal. Recent researchers have focused on green routes for the synthesis and production of nanoparticles [8]. Green synthesis is one of the simplest and easiest methods for synthesis of NPs. The advantage of producing NPs by this method is that it is easy, fast, cost effective, and flexible. The physicochemical properties of NPs, such as size, agglomeration status in liquid and surface charge, play an important role in the ultimate interactions of the NPs with target cells.

Faheem et al. [2] synthesized $\mathrm{CuO}$ NPs using Abutilon indicum leaf extract, has acted as a fuel, and finally reported the antioxidant activity and anti-microbial activity of $\mathrm{CuO}$ NPs. About $1000 \mu \mathrm{g}$ of $\mathrm{CuO}$ NPs produced better anti-oxidant effects similar and comparable to that of the standard anti-oxidant BHT. The results of anti-bacterial activity of $\mathrm{CuO}$ NPs produced higher activity than the standard drug Ampicillin against Klebsiella sp., Bacillus subtilis, Escherichia coli and Staphyloccus aureus.

In recent days, organic dyes are the major pollutant in our environments. It is not easy to remove from dye-bearing waste waters. Presently varieties of method are available for the removal of dyes from contaminated water. But scientists and researchers focused nanoparticles which has excellent property to absorb the dyes from the polluted environment [9]. Pourahman [10] reported that Silver sulfide nanoparticles ( $\mathrm{Ag}_{2} \mathrm{~S}$ NPs) can efficiently degrade methylene blue in aqueous solution. Metal oxide nanoparticles act as a good photocatalyst, specifically $\mathrm{CuO}$ NPs find a special place in photo catalysis under sunlight. Sundaramurthy and parthiban [11] reported the $\mathrm{CuO}$ NPs has excellent efficiency to degrade methylene blue under solar irradiation. The green-synthesized $\mathrm{CuO}$ NPs were confirmed as efficient catalysts with enhanced rates of textile dye decolourisation.

The $\mathrm{CuO}$ NPs can also suppress cell viability using several mechanisms such as apoptosis and necrosis. Cell suicide mechanism which controls cell numbers is apoptosis. Two types of pathway are involved in apoptosis. Nagajyothi et al. [12] reported, $\mathrm{CuO}$ NPs showed a great cytotoxic effect on $\mathrm{HeLa}$ cells in a concentration dependent manner. Finally, $\mathrm{CuO}$ NPs have being used as photocatalyst, anti-microbial agent and better anti-cancer compound with minimal side effects. $\mathrm{CuO}$ NPs can also act as efficient anti-bacterial agent when incorporated in coatings, plastics, textiles, etc.

\section{References}

1. Ameer A, Arham SA, Oves M, et al. Size-dependent antimicrobial properties of $\mathrm{CuO}$ Nanoparticles against Gram-positive and Gram-negative bacterial strains. Int J Nanomedicine. 2010;7:3527-35.

2. Faheem I, Sammia S, Shakeel AK, et al. Green synthesis of copper oxide nanoparticles using Abutilon indicum leaf 
Citation: Bhuvaneshwari V, Vaidehi D, Logpriya S. Green synthesis of copper oxide nanoparticles for biological applications. Microbiol Curr Res. 2018;2(1):5-6.

extract: Antimicrobial, antioxidant and photocatalytic dye degradation activities. Trop $J$ of Phar Res. 2016;16:743-53.

3. Yallappa S, Manjanna J, Sindhe MA, et al. Microwave assisted rapid synthesis and biological evaluation of stable copper nanoparticles using $\mathrm{T}$. arjuna bark extract. Spectrochim Acta Mol Biomol Spectrosc. 2013;110:108-15.

4. Seigneuric R, Markey L, Nuyten DSA, et al. From nanotechnology to nanomedicine: applications to cancer research. Curr Mol Med. 2010;10:640-52.

5. Liu Z, Kiessling F, Gatjent J. Advanced nanomaterials in multimodel imaging: design, functionalization, and biomedical applications. J Nanomater. 2010;894003.

6. Kwak K, Chongyoup K. Viscosity and thermal conductivity of copper oxide nanofluid dispersed in ethylene glycol. Korea-Aust Rheol j. 2005;17:35-40.

7. Stoimenov PK, Rosalyn L, Klinger, et al. Metal oxide nanoparticles as bactericidal agents. 2002;18:6679-86.

8. Sankar R, Manikandan P, Malarvizhi V, et al. Green synthesis of colloidal copper oxide nanoparticles using Carica papaya and its application in photocatalytic dye degradation. Spectrochim Acta Mol Biomol Spectrosc. 2014;121:746-50.

9. Manoranjan B, Gitisudha G. Inquiring the photocatalytic activity of cuprous oxide nanoparticles synthesized by a green route on methylene blue dye. Int $\mathrm{J}$ Ind Chem. 2016; 7:157-66.
10. Pourahmad A. Ag2S nanoparticle encapsulated in mesoporous material nanoparticles and its application for photocatalytic degradation of dye in aqueous solution. Supperlattices Microstruct. 2012;52:276.

11. Sundaramurthy N, Parthiban C. Biosynthesis of copper oxide nanoparticles using pyrus pyrifolia leaf extract and evolve the catalytic activity. Int Res J Eng Technol. 2015;2:332-8.

12. Nagajyothi PC, Muthuraman P, Sreekanth TVM, et al. Green synthesis: in-vitro anticancer activity of copper oxide nanoparticles against human cervical carcinoma cells. 2016;1-11.

\section{*Correspondence to:}

Bhuvaneshwari V

PG and Research Department of Biotechnology,

Kongunadu Arts and Science College,

Coimbatore-641029,

Tamil Nadu,

India

Tel: $+91-9894107126$

E-mail: bhuvana_bt@yahoo.co.in 